\title{
Use Spreading Activation to Model E2 Level in Representation Redescription
}

\author{
Xixu Fu and Xizhang Gong \\ Institute of Information and Education Technology, Shanghai Ocean University, Shanghai, China
}

\begin{abstract}
Representational redescription is an important theory which describes conceptualization as an implicit to explicit process. This paper analyzed stages in the description in two dimensions on structural formation and generalization respectively. Focus on the structural formation dimension, this paper advanced a hypothesis based on spreading activation theory. The hypothesis was used to explain the acquisition of express solving multiplication problem.
\end{abstract}

Keywords-representational redescription; spreading activation; knowledge acquisition; multiplication

\section{INTRODUCTION}

As a conciliation of Fordor's nativism and Piaget's constructivism, representational redescription is an important theory focus on the development of concepts in human mind [4][9]. The theory described concept development in the dimension of explicitness. Higher level of knowledge is represented in a more explicit level. The development of knowledge in mind is divided into four levels from I to E3. Features of each level are introduced in Kamiloff-Smith's book in detail [8]. The description in the book is in the language of psychology but hard to formalize.

According to the description of representation levels in representational redescription theory, the representations can be described in two dimensions. One is connectivity which describes the structure of concepts the other is generalization which describe the categorizing of them. All levels in representational redescription theory can be explained in the two-dimension model. Level I is a simple initializing process. Level E1 is a more generalized representation level than level I. Level E2 is a level of connecting. Concepts are connected to each other and problem solving becomes more efficient. Level E3 is an advanced level in which concepts are refined and fit for oral presentation.

Study of level E2 is the topic of this paper. This level is mainly about connection and reference. Because micro fields and concepts have been formed in this level, traditional method do not work in the simu lation of this level. Spreading activation theory can deal with this problem well. Spreading activation model is a famous theory about reasoning in human memory based on ACT-R theory Anders on [1]. The theory can explain the nature of human association and some problem solving methods. It is efficient to use this theory to model knowledge systems [5].

This paper set up a model for level E2 of representational redescription theory using spreading activation theory. The study focus on the solving of complicated multiplication problem of children who have grasped simple multiplication. This example is better than study of infants as an example for evolving of existing knowledge structure.

The paper first introduced representation redescription theory and spreading activation theory to explain the motivation and related work. Then a connection based model was set up for further study. After that, complex multiplication behavior of human was studied. Further details were simulated on the computer to testify the model. At last some discussions were carried out on spreading activation and representational redescription theory.

\section{REPRESENT ATIONAL REDESCRIPTION AND RELATED WORK}

\section{A. Representational Redescription and Related Studies}

Representational redescription is a hypothesis about the human knowledge acquisition process. Representational redescription was described in three stages as follows [8]:

In the first stage, children focus on outer information from the environment. Learning in this stage is materially driven. Children focus on outer materials to form representational associations in all micro fields. It is provided that representational associations are stable in representation and not related to outer materials. Once new stable representation established, they are field-specially associated with other existing materials and have little effect on the representation. In other words, the representational associations do not change the level of representation. The result of the first stage is that all fields that reach this level gain coherent success in behaviors which is socalled behavioral grasp.

The next stage is an internally driven stage. In this stage, children do not focus on outer materials. Motion in the inner system takes effects. Inner representation becomes the focus of evolving. In the second stage, children's present representation becomes superior to the information in input materials. New mistakes and rigidness emerge due to ignorance of the outer environments. Although the evolving process can decrease the success rate of behaviors, it is progress at representation level.

Finally, at the third stage, inner representation and outer materials become compromised and inner and outer controls reached a balance. This produces higher level of representation.

Four levels of representation were advanced corresponding to the stages. Representations in the implicit (I) level are 
merely simple repetitions of acquired scenarios. They cannot adapt to any changes in problems or scenarios. Representations in the first explicit (E1) level can adapt to environmental changes. They have some generalize ability. Representations in the second explicit (E2) level can represent abundant relations of concepts, but cannot be used for oral presentations. Representations in the third explicit (E3) level can access to the consciousness. They are concise enough for oral presentations. Many psychological documents discussed representation redescription process in children's different behaviors $[2][6][10][11]$. Recent reports have indicated the existence of level I and E1 representation [2].

Some studies have analyzed the psychological aspects and setup of representation redescription in detail using UML and object-oriented language [7]. However, these papers are mainly simple descriptions based on psychological analysis. The essence of representation redescription has not been mentioned in these works. Although the generalization essence evolution had been advanced based on the learning of concept of round, the model advanced is too simple to explain the whole redescription process [13].

\section{B. Spreading Activation as a Connectionnist Model}

Spreading activation theory is a theory about knowledge in men's memory [12]. John R. Anderson advanced ACT-R frame to describe spreading activation theory [1]. The theory has been wildly used in recommend systems and semantic models [3]. The theory is promising to describe dynamic actions. A model representing the interactions has been advanced in recent work [5].

\section{PROBLEM AND MOTIVATION}

\section{A. Analysis of Levels in Representation Redescription}

Kamiloff-Smith described representation redescription in four levels. Focus on one micro field, it easy to see a concept is formed in two directions. One is structural, the other is about generalization. The evolution process is shown in Fig. 1.

Labels are associated to objects in real scenario to generate level I representation first. Obviously, the representation is just a correspondence to real world. It is not flexible at all. In the next stage, categories are generated by redescription and induction, the result is level E1 representation. It is easy to comprehend the representation's flexibility on object change. This is a level more generalized than level I. The next level is level E2. It is the main topic of this paper. In this level, micro fields are connected to each other, express path can be established for special situations. It is a higher level than level E1 in the connectivity dimension. Level E3 is a brief representation of frequently activated nodes.

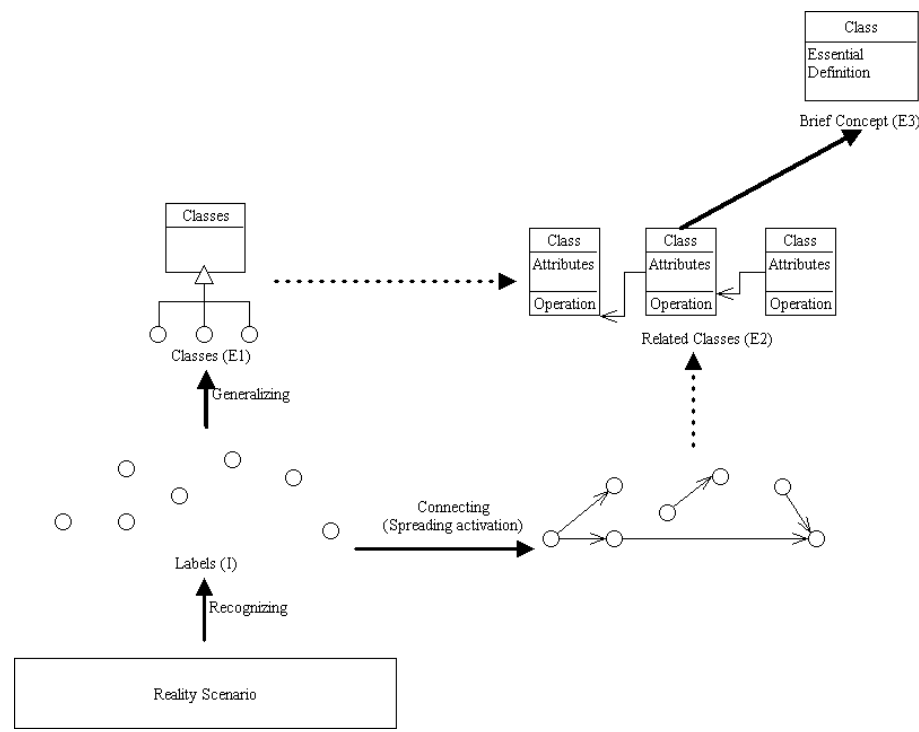

FIGURE I. LEVELS OF REPRESENT ATIONAL REDESCRIPTION DESCRIBED IN TWO DIMENSIONS

\section{B. Complicated Multiplication: A Good Example for E2 Level}

Solving of regular complicated multiplication is a good example of E2 level. Take equation (1) as an example.

$$
12138 \times 16 \times 25 \times 125 \times 4 \times 25 \times 2=?
$$

It is time consuming to multiply the numbers one by one. However, the problem turns easy when equation (2) considered.

$$
12138 \times 2^{4} \times 5^{2} \times 5^{3} \times 2^{2} \times 5^{2} \times 2=?
$$

Consider $2 \times 5=10$, the answer become clear as shown in equation (3).

$$
12138 \times 10^{7}=121380000000
$$

\section{STUDY CHILDREN'S SOLVING OF COMPLICATED MULTIPLYING PROBLEMS}

Experiments are carried out on twenty children who are 10 to 12 years old. Children in this age can master multiplication but can not master more complicated concepts such as power and exponent. In the first experiment, they were asked to calculate equation (1) without guidance. In the second experiment, the same children were asked to solve equation (1) again after knowing the effective way. In the third experiment children were asked to solve equation (4) without guidance.

$$
76239 \times 32 \times 25 \times 25 \times 8 \times 25 \times 25=?
$$

We recorded time and methods children solve the problem. Then we categorized the children by the method they used. 
The results of the experiments are shown in table 1. Children use effective method were labeled category A. Children use traditional method were labeled category B. The number of children in category $A$ is noted as $N_{A}$. The number of children in category $B$ is noted as $N_{B}$. The average time consumed by two categories are noted as avgT(A) and avgT(B) respectively. The standard deviation of time consumed were noted as $\operatorname{dev} \mathrm{T}(\mathrm{A})$ and $\operatorname{dev} \mathrm{T}(\mathrm{B})$ respectively.

TABLE I. TIME CONSUMING OF CALCULATION (UNIT OF TIME: SECOND)

\begin{tabular}{|l|l|l|l|l|l|l|}
\hline Expe riment & $\mathbf{N}_{\mathbf{A}}$ & $\operatorname{avgT(A)}$ & $\operatorname{devT(A)}$ & $\mathbf{N}_{\mathbf{B}}$ & $\operatorname{avgT(B)}$ & $\operatorname{devT(B)}$ \\
\hline 1 & 1 & 463 & 0 & 19 & 1263 & 316 \\
\hline 2 & 20 & 71 & 11 & 0 & $/$ & $/$ \\
\hline
\end{tabular}

\section{COMPUTER METHOD AND MODEL}

\section{A. Representation}

Spreading activation model is a connectional method. Nodes and connections are basic elements of this method. A concept can be regarded as a node. Variables and operations in concepts can be regarded as nodes too. A node is represented in two parts, one is input, the other is activation function. A node is connected to another node if and only if it is used as an input of the node. The weight of the edge between the nodes is the time consuming of the execution. It can be decided only when the nodes activated. The representation of activation of factorize function is shown in Fig. 2.

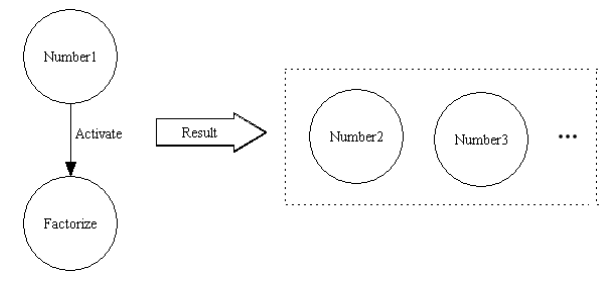

FIGURE II. ACTIVATION OF FACT ORIZE FUNCTION

Number and Factorize are two nodes of the scenario. Once the number is used as the parameter of factorize function, the Factorize node is activated. The resulted nodes replaced the original nodes in the scenario according to the definition of activation function.

The model is dynamic. It can be represented as snapshots of states. We use $\mathrm{N}$ to represent all node in a state as a set. The state is represented as a $\mathrm{N} \times \mathrm{N}$ matrix. Weights are stored in the matrix.

\section{B. Spread activation to Solve Problem}

Soaking from nodes can get a fast solution of a problem. Take the complicated multiplication in equation 1 as an example, the soaking process is shown in Fig. 3. For the convenience of representation and reading, node multiply and factorize are abbreviated to lines and arrowed lines respectively.
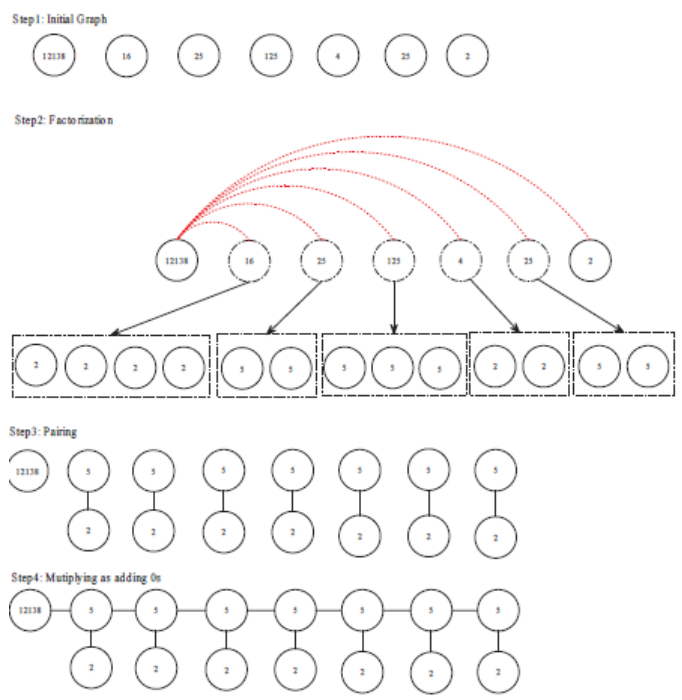

FIGURE III. SPREADING ACTIVATIONT OSOLVE MULTIPLICATION PROBLEM

Numbers to be multiplied are regarded as initial nodes. If two nodes are multiplied, they are connected to each other to form a solution. However, it is difficult to compute the multiplications directly. For example, computing $12138 \times 25$ is quite time consuming. So the factorize functions are activated first. The $5 \mathrm{~s}$ and $2 \mathrm{~s}$ are multiplied first to form $10 \mathrm{~s}$. At last, all $10 \mathrm{~s}$ are multiplied to node 12138 to get the final result. The process can be described in the algorithm shown in Fig. 4.

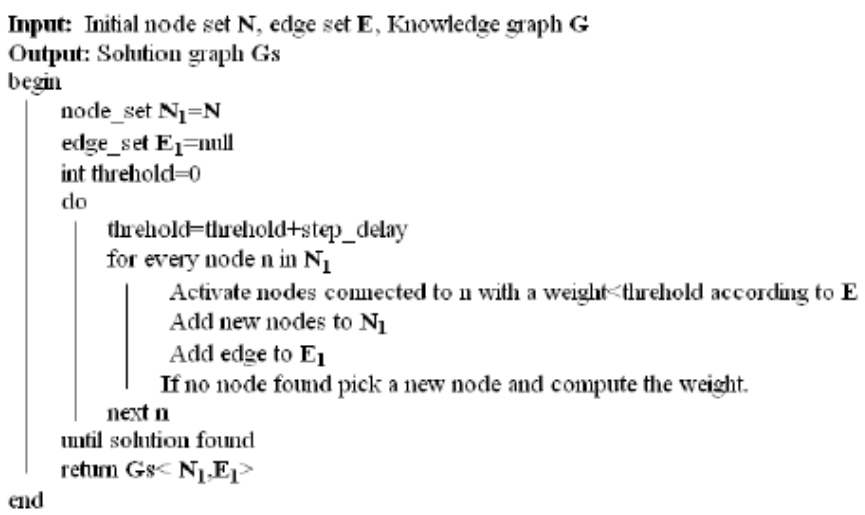

\section{FIGURE IV. SPREADING ACTIVATION ALGORITHM}

\section{Redescribe to Learn}

The learning process can be divided into unsupervised learning and supervised learning. In supervised learning, frequent paths are acquired in the training of repeating right instances. Take complicated multiplication as an example, connection $5 \times 2=10$ is reinforced and given a low weight. Children can use the rule immediately when new problem occurs. In unsupervised learning, the spreading activation algorithm is applied to get the right weight. Because the large amount of candidate connections, the process may be slow first. The learning process become fast after the gaining of right weight. 


\section{EXPERIMENTS AND RESULTS}

Experiments on unsupervised learning collect the running time of equation (1). The initial setting of activation weight is shown in table 2.

\section{TABLE II. WEIGHT OF ACTIVATIONS}

\begin{tabular}{|l|l|l|l|l|}
\hline $\begin{array}{c}\text { Node1 } \\
\text { (calculus) }\end{array}$ & $\begin{array}{l}\text { Node2 } \\
\text { (input) }\end{array}$ & $\begin{array}{c}\text { Node3 } \\
\text { (input) }\end{array}$ & Weight & O utput \\
\hline times & 2 & 5 & 1 & 10 \\
\hline times & $\mathrm{x}$ & $10^{\mathrm{n}}$ & 1 & $\mathrm{x} 000 \ldots$ \\
\hline factorize & 25 & & 1 & 5,5 \\
\hline factorize & 4 & & 1 & 2,2 \\
\hline factorize & 16 & & 2 & $2,2,2,2$ \\
\hline factorize & 125 & & 2 & $5,5,5$ \\
\hline
\end{tabular}

Weights of normal multiplications not shown in the table are related to the digit capacity of the numbers. They are calculated use equation (5).

$$
\text { Weight }=\log (\text { nu mber } 1) \times \log (\text { nu mber } 2) \times 10
$$

Total time used to solve a problem is defined as the total weight of edges activated. Table 3 shows the total time of different turning time.

\section{TABLE III. WEIGHT OF ACTIVATIONS}

\begin{tabular}{|l|l|}
\hline Step Delay & Total Time \\
\hline 1 & 22 \\
\hline 10 & 22 \\
\hline 100 & 121 \\
\hline 1000 & 1650 \\
\hline
\end{tabular}

To show the result of supervised training, the right result of equation (1) is used to train a model for solving equation (4). The evaluation criterion is the same as in the unsupervised learning. The result shows the soaking can reach the best result quickly.

\section{CONCLUSION}

This paper simulated level E2 representation of representational redescription theory using spreading activation model. The experiments on children proved the soaking process. The simulation on computer described the detailed process of knowledge referencing. The model is explained level E2 in the representational redescription theory in a soaking based way.

\section{ACKNOWLEDGMENT}

We thank the project with granted number A2-0203-17100331 for their support.

\section{REFERENCES}

[1] Anderson, J. R. and Pirolli, P. L., "Spread of activation," Journal of Experimental Psychology:Learning, Memory, and Cognition, vol. 10, pp. 791-798, 1984

[2] Cheung C.N. and Wong, W.C., "Understanding conceptual development along the implicit-explicit dimension: Looking through the lens of representational redescription model," Child Development, vol. 82, pp. 2037-2052, 2011.
[3] Crestani F., "Application of spreading activation techniques in information retrieval," Artificial Intelligence Review, vol. 11, pp. 453482, 1997.

[4] Fodor J. A., "Precis of the modularity of mind," THE BEHAVIORAL AND BRAIN SCIENCES, vol. 8, pp. 1-42, 1985.

[5] Fu X. and Wei H., "Problem solving by soaking the concept network," Computer Science and Information Systems, vol. 8, pp. 761-778, 2011.

[6] Horst Krist, T. S., Holger Horz, "Children's block balancing revisited: No evidence for representational redescription," Swiss Journal of Psychology, vol. 64,pp. 183-193,2005.

[7] Hui Wei, W. H., and Chen, Y., "Uml-based representational redescription of concept development," In Proceedings of the sixth international conference on machine learning and cybernetics, pp. 131139, 2007.

[8] Kamiloff-Smith A., "Beyond modularity: A developmental perspective on cognitive science," USA: Massachusetts Institute of Technology, 1992.

[9] Kamiloff-Smith A., "Precis of beyond modularity: A developmental perspective on cognitive science.," Behavioral and Brain Science, vol. 17, pp. 693-745, 1994.

[10] Puche-Navarro R., "From implicit to explicit representation in children's response to pictorial humor.," International Journal of Behavioral Development, vol. 33, pp. 543-555, 2009.

[11] Sarah Critten, K. P., Steffler, D., "Spelling development in young children: A case of representational redescription?" Journal of Educational Psychology, vol. 99, pp. 207-220, 2007.

[12] Warren, R. E., "T ime and the spread of activation in memory.," Journal of Experiment al Psychology: Human Learning and Memory, vol. 3, pp. 458-466, 1977.

[13] Wei, H. and Fu, X., "The evolving of concept system based on representation redescription.," In Proceedings of 2009 international conference on machine learning and computing, pp. 117-122, 2009. 